\title{
Propiedades físicas y dinámica de los combustibles forestales en un bosque de encino
}

\section{Physical properties and dynamics of forest fuels in an oak forest}

\author{
Juan Leyver Martínez Villa', Dante Arturo Rodríguez-Trejo'* Ma. Amparo Máxima Borja-de la Rosal \\ y Gabriel Arcángel Rodríguez Yam!
}

'Universidad Autónoma Chapingo. División de *Autor de correspondencia. dantearturo@yahoo.com

Ciencias Forestales. Chapingo, Estado de México,

México.

\section{RESUMEN}

Se evaluaron y analizaron propiedades físicas de combustibles forestales de importancia en la estimación del comportamiento del fuego y la dinámica de los combustibles durante la temporada de incendios forestales en un encinar del Edo. de México. Se estimó: i) densidad básica y humedad de los combustibles por tiempo de retardo (TR); ii) carga de combustibles leñosos por tres variantes de método; y iii) dinámica de combustibles forestales. Las densidades básicas de los materiales leñosos decrecieron a mayores tiempos de retardo: $1 \mathrm{~h}=0.585 \mathrm{~g} \mathrm{~cm}^{-3}, 10 \mathrm{~h}=0.478 \mathrm{~g} \mathrm{~cm}^{-3} \mathrm{y} 100 \mathrm{~h}=0.412 \mathrm{~g} \mathrm{~cm}^{-3}$. La carga de los combustibles leñosos, estimada con densidades básicas de los materiales recolectados, fue la menor: $11.6 \%$ menos con respecto al valor obtenido usando densidades básicas de coníferas y $43 \%$ menos al usar la densidad básica de encinos referida en la bibliografía. La incorporación de materiales fue de $40.532 \mathrm{Mg} \mathrm{ha}^{-1}$ durante la temporada de incendios; para hojarasca correspondió 62.9\% $(25.491 \mathrm{Mg}$ ha-1) y para leñas $18.8 \%$ (7.603 Mg $\mathrm{ha}^{-1}$ ). La tasa de descomposición en hojarasca fue 0.0827 y en leñas 0.0861 y 0.0404 , para $1 \mathrm{~h}$ y $10 \mathrm{~h}$ TR, respectivamente. La carga acumulada al final de la temporada incrementó $205 \%$ en ramillas de 1 h y 10 h TR, mientras que la hojarasca lo hizo en $58.7 \%$.

PALABRAS ClAVE: densidad básica; descomposición; incendios forestales; incorporación de detritos; tiempo de retardo.

\section{ABSTRACT}

In this work physical properties relevant in estimations of fire behavior and the forest fuels dynamics in an oak forest in the Estado de Mexico, Mexico were analyzed and evaluated. The evaluation was conducted along the forest fires season. It was estimated: i) the basic density and moisture content of woody fuels by time lag; ii) the forest fuel load in three ways; and iii) the forest fuels dynamics. The basic densities of the woody fuels in the study site followed a decreasing tendency: $1 \mathrm{~h}=0.585 \mathrm{~g} \mathrm{~cm}^{-3}, 10 \mathrm{~h}=0.478 \mathrm{~g} \mathrm{~cm}^{-3}$, and $100 \mathrm{~h}=0.412 \mathrm{~g} \mathrm{~cm}^{-3}$. The woody fuel load estimated with basic densities from the collected material was the lowest: $11.6 \%$ less than the value obtained using conifer basic densities, and $43 \%$ less than using the basic density of oaks from bibliography. The incorporation rate of fuels was equal to $40.532 \mathrm{Mg} \mathrm{ha}^{-1}$, along the fire season, with $62.9 \%\left(25.491 \mathrm{Mg}\right.$ ha $\left.{ }^{-1}\right)$ for leaf litter, and $18.8 \%\left(7.603 \mathrm{Mg} \mathrm{ha}^{-1}\right)$ for woody fuels. The decomposition rate (k) during the fire season was of 0.0827 for leaf litter, and for woody fuels 0.0861 for $1 \mathrm{~h}$ time lag, and 0.0404 for $10 \mathrm{~h}$ time lag. Biomass load added to the forest floor at the end of the season increased to an accumulated load of $205 \%$ for $1 \mathrm{~h}$ and $10 \mathrm{~h}$ time lag for branches, while for the leaf litter was $58.7 \%$.

KEYWORDS: basic density, decay, forest fires, litter decomposition, time lag.

\section{INTRODUCCIÓN}

Como parte del manejo del fuego, es necesario el conocimiento de las propiedades físicas de los combustibles forestales, ya que se trata de variables necesarias para modelar el comportamiento del fuego y sus efectos potenciales (Sandberg, Ottmar y Cushon, 2001), como son las emisiones. Ejemplos de estas propiedades son: disposición de los materiales combustibles (superficial, subterránea o aérea), continuidad horizontal y vertical, peso, estado de descomposición, tiempo de retardo, carga, tamaño del combustible, relación superficie/volumen, compactación, contenido de humedad y densidad básica, entre otras. 
La importancia de determinar la densidad básica de los materiales combustibles estriba en obtener estimaciones más precisas de la carga, comportamiento y efectos del fuego, tanto durante incendios forestales como en quemas prescritas. El método de Brown (1974), fundamentado en Van Wagner (1968), para combustibles leñosos, es el más usado en el mundo. Este método fue publicado usando densidades básicas de combustibles leñosos de coníferas de Estados Unidos.

La densidad básica es una de las propiedades físicas que está directamente relacionada con la dureza, el peso, la resistencia al impacto y a la abrasión de la madera (Ordóñez-Díaz et al., 2015). En los incendios forestales, la densidad básica juega un papel importante al relacionarse positivamente con la conductividad térmica (Byram, Fons, Sauer y Arnold, 1952). Las maderas densas, a pesar de que tardan más en arder, una vez que lo logran lo hacen con mayor intensidad que las menos densas. Esto se debe a que los materiales de baja densidad tienen menores conductividades térmicas (Hyde, Smith, Ottmar, Alvarado y Morgan, 2011) y a que cuentan con una menor masa a ser consumida por unidad de volumen (Rodríguez-Trejo, 2015). Es esencial entender los efectos de la descomposición en las propiedades físicas de los combustibles leñosos y su significancia en la combustión, pues con la descomposición se experimentan cambios físicos y químicos que afectan el consumo por el fuego (Hyde et al., 2011).

La dinámica de combustibles forestales se refiere al proceso de incorporación y descomposición de los materiales que potencialmente pueden entrar en ignición. El balance entre la entrada de materia orgánica y la descomposición heterotrófica influye en la dinámica del carbono y en los ciclos de nutrientes (Karberg, Scott y Giardina, 2008). Esto es importante en los bosques debido a que los requerimientos de nutrientes en su mayoría son satisfechos por la descomposición de la materia orgánica (Landsberg y Gower, 1997).

La descomposición de los combustibles forestales depende de las condiciones atmosféricas, la composición química del material, los organismos descomponedores y las características del suelo (Álvarez-Sánchez y Harmon, 2003). Esta complejidad hace que las tasas de descomposición varíen en los diferentes ecosistemas y regiones del mundo. En ecosistemas estacionales, como los bosques templados de encino, la tasa de descomposición varía a través de las estaciones del año y es durante la época de lluvias cuando se presenta la mayor tasa de descomposición (Álvarez-Sánchez y Harmon, 2003; MartínezYrízar y Sarukhán, 1993).

Los estudios de descomposición de detritos en México, se han hecho principalmente en hojarasca, a través del método de masas remanentes. Poca atención ha sido dada a la degradación de los materiales leñosos. La magnitud del $\mathrm{CO}_{2}$ que retorna a la atmósfera, a partir de los restos leñosos en descomposición, es comparable con la combustión global actual de los combustibles fósiles (Le Quéré et al., 2013).

Propiedades físicas como el grosor de los materiales leñosos retrasan la descomposición; los materiales gruesos son más resistentes a la pudrición que los delgados (Müller-Using y Bartsch, 2009), acumulándose en el piso forestal por muchos años. La importancia de la restauración de los regímenes naturales del fuego en los bosques radica en que forman parte de la dinámica de los combustibles, ya que la mortalidad de árboles y la caída de ramas, así como las actividades de aprovechamiento forestal, aumentan la carga de restos leñosos, mientras que el fuego los remueve y transforma (Tainter y McMinn 1999).

Los estudios sobre dinámica de combustibles se han conducido anualmente, casi no se han enfocado a la temporada de incendios. Durante ella las cargas y su influencia en el comportamiento del fuego, pueden variar.

\section{OBJETIVOS}

Con base en lo anterior, los objetivos del presente trabajo fueron: cuantificar tres propiedades físicas de combustibles forestales (carga, densidad básica de los materiales leñosos y contenido de humedad) en un bosque de encino, durante una temporada de incendios forestales, además de analizar la dinámica de combustibles forestales, a lo largo de tal temporada.

\section{MATERIALES Y MÉTODOS}

\section{Área de estudio}

El área de estudio se ubica en la vertiente del río "Los Hornos", perteneciente a la cuenca del Río Texcoco, que atraviesa la comunidad de San Pablo Ixayoc en el oriente del Estado de México (Fig. 1). Se caracteriza por un relieve accidentado. El clima es templado subhúmedo con lluvias en verano, la precipitación promedio anual va de $620 \mathrm{~mm}$ a $770 \mathrm{~mm}$ de 15.9 
${ }^{\circ} \mathrm{C}$ (Adame-Martínez, Oropeza-Mota, Martínez-Méndez, Santoyo-Cortés y Ramírez-Guzmán, 2000).

El dosel forestal en el área de investigación está dominado por Quercus crassipes Humb. \& Bonpl., Q. rugosa Neé y Q. laeta Liebm., con menor presencia de Q. laurina Humb. \& Bonpl. Además, se encontró el encino arbustivo Q. frutex Trel. en los espacios abiertos de las laderas con exposición tendiente a sur en las cañadas. La identificación se hizo con las claves dicotómicas de Romero-Rangel, Rojas-Zenteneo y Aguilar-Enríquez (2002), para encinos del Estado de México. Quercus rugosa predomina sobre laderas secas con exposición suroeste. Las laderas húmedas con exposición noreste tenían una mezcla de las otras tres especies.

\section{Estimación de carga inicial}

El levantamiento de datos de la carga de combustibles leñosos se realizó con la metodología de intersecciones planares de Brown (1974), empleando cuatro conglomerados de muestreo como los referidos por Flores-Garnica et al. (2008) y detalles del método proporcionados por Morfín-Ríos et al. (2012). En campo se usaron las siguientes longitudes de línea por tiempo de retardo (TR): 2 m (1 h TR), 4 m (10 h TR) y 12 m (100 h y 1000 h TR). La disposición de las unidades de muestreo se proporciona en la figura 2.

La determinación de la carga de combustibles finos (hojarasca y capa de fermentación) se obtuvo por peso anhidro a partir de unidades de muestreo cuadradas de $30 \mathrm{~cm} \times 30 \mathrm{~cm}$ (tres por conglomerado, 12 en total, superficie de muestreo de $1.08 \mathrm{~m}^{2}$ ). La carga de los combustibles vivos se determinó por peso seco del material recolectado, en cuadrados de $1 \mathrm{~m}^{2}$ (uno por conglomerado, 4 en total, superficie de muestreo igual a $4 \mathrm{~m}^{2}$ ). Ambos resultados, en gramos por metro cuadrado, se extrapolaron a megagramos por hectárea. La estimación de la carga de material leñoso se hizo de tres formas: 1) con las fórmulas de Brown (1974) sin ajustar por la densidad de la madera; 2) las de Van Wagner (1982) usando la densidad básica promedio señalada en bibliografía para las especies del área; y 3) las de Van Wagner (1982) empleando densidad básica determinada en laboratorio a partir de materiales recolectados en el encinar.

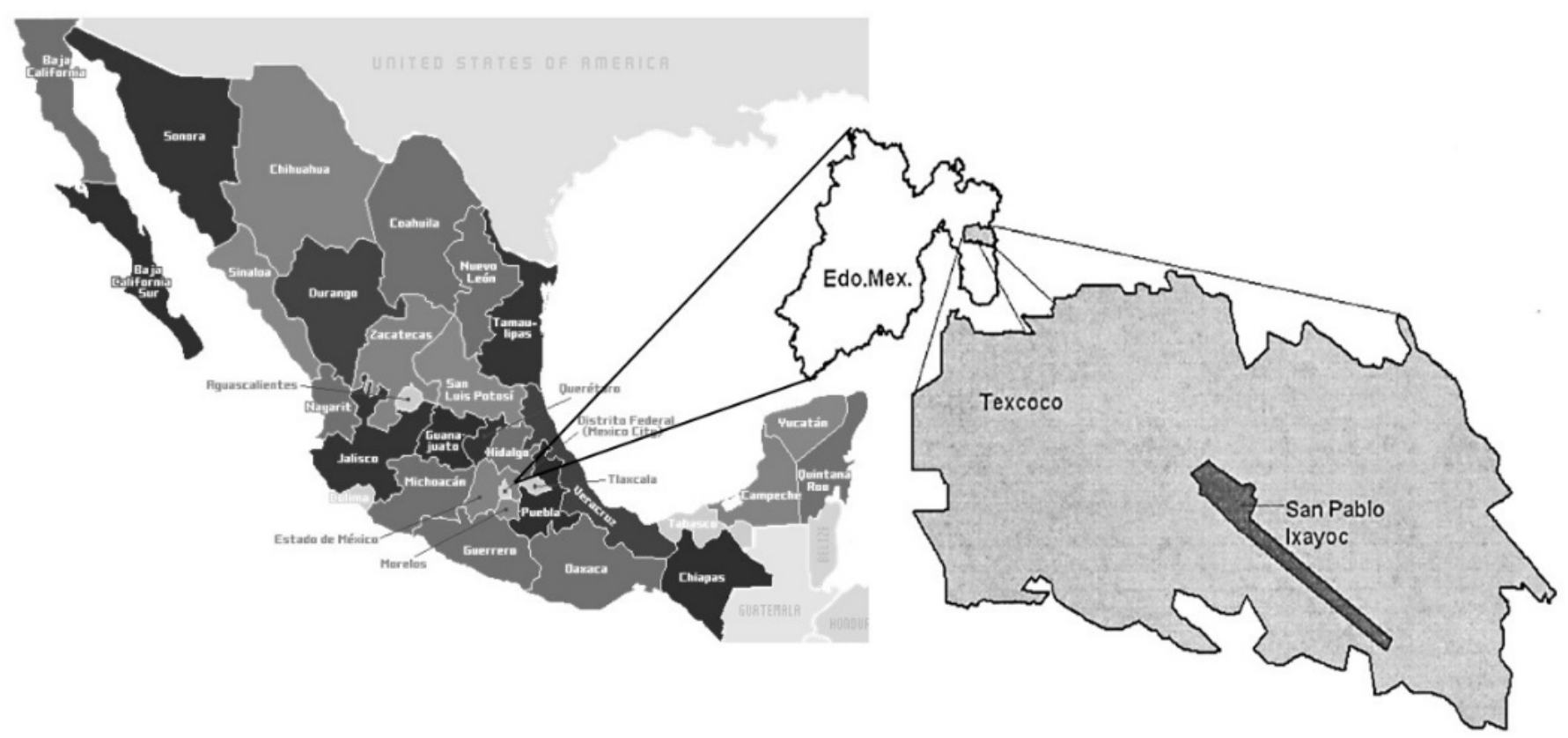

Figura 1. Localización de San Pablo Ixayoc (Instituto Nacional de Estadística, Geografía e Informática [Inegi], 2005). 


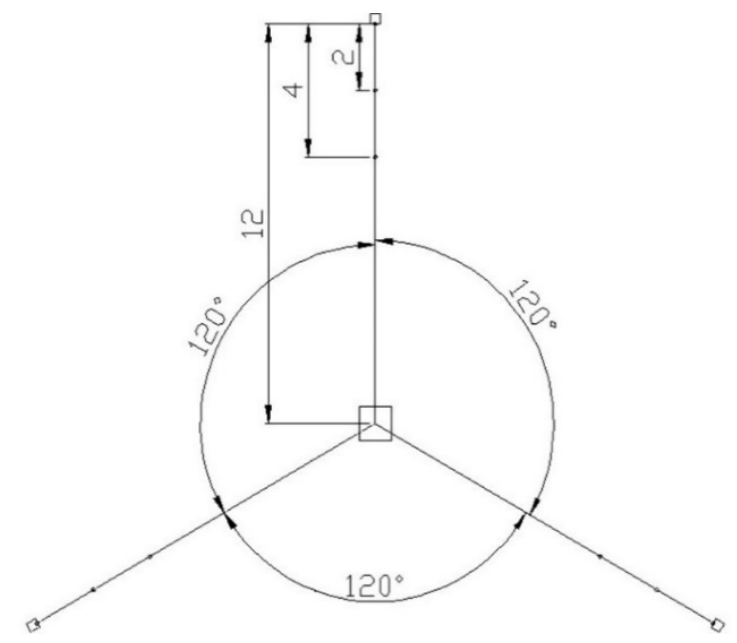

FIGURA 2. Disposición de las unidades de muestreo en el conglomerado.

La línea superior fue orientada al norte.

\section{Densidades básicas}

La determinación de la densidad básica de combustibles leñosos se llevó a cabo por TR. Fueron empleadas 270 ramillas de 1 h TR, 93 ramillas de 10 h TR y 18 ramas de 100 h TR. Estos materiales fueron recolectados de los conglomerados de muestreo para estimación de carga. La densidad básica se determinó para cada tiempo de retardo, a partir del peso anhidro entre el volumen, obtenido por el método fundamentado en el principio de Arquímedes.

Se llevó a cabo una revisión bibliográfica con el fin de recopilar las densidades básicas estudiadas para las distintas especies presentes en el encinar de estudio. De ellas se obtuvo una densidad promedio $\left(0.665 \mathrm{~g} \mathrm{~cm}^{-3}\right)$. Este valor se supuso para los combustibles 100 h y 1000 h TR y se ajustó con 20\% más para la densidad básica de los combustibles de 1 h y 10 h TR, con base en Brown (1974).

\section{Contenido de humedad de los combustibles leñosos}

La humedad de los combustibles leñosos se obtuvo para tres momentos de la época de estiaje: 20 de noviembre de 2015, 20 de febrero y 20 de mayo de 2016. Para la selección del material leñoso, se instaló un conglomerado de muestreo, igual al empleado para la estimación de la carga de combustibles leñosos. A cada momento de evaluación le correspondió una línea de intersección, empezando por la línea norte y siguiendo en dirección a las manecillas del reloj. El material leñoso que intersectó la línea fue recolectado y pesado en campo a las $14 \mathrm{~h}$ 00 , posteriormente se trasladó a laboratorio en sobres de papel manila o bolsas de papel, según el tamaño del material, donde fue secado en horno $\left(75^{\circ} \mathrm{C}\right)$ hasta obtener su peso seco.

El modelo usado para estimar el contenido de humedad $(\mathrm{CH})$, fue:

$$
\mathrm{CH}=\frac{\left(\mathrm{P}_{\mathrm{f}}-\mathrm{P}_{0}\right)}{\mathrm{P}_{0}}(100)
$$

donde:

$\mathrm{P}_{\mathrm{f}}=$ peso en fresco $(\mathrm{g})$

$\mathrm{P}_{0}=$ peso anhidro $(\mathrm{g})$

\section{Estimación de la incorporación de material combustible}

La evaluación de la incorporación de los materiales combustibles en el encinar se realizó con 12 trampas de hojarasca de $1 \mathrm{~m}^{2}$, tres para cada una de las siguientes combinaciones de exposición y cobertura: sitio I (ladera húmeda, dosel cerrado), sitio II (ladera húmeda, dosel abierto), sitio III (ladera seca, dosel abierto) y sitio IV (ladera seca, dosel cerrado). Las trampas fueron elaboradas con bastidores de madera y tela de tul, instaladas el 5 de noviembre de 2015. El material atrapado por las trampas fue recolectado en dos ocasiones, febrero de 2016 y mayo del mismo año. En el laboratorio fueron separados por tipo de material. La determinación de la carga incorporada por tipo de combustible se obtuvo dividiendo el peso anhidro de cada material (g) por el área de la trampa de hojarasca $\left(1 \mathrm{~m}^{2}\right)$, y extrapolado a megagramos por hectárea. La suma de la biomasa de los dos trimestres, según el tipo de material combustible, fue la biomasa total incorporada en un sitio durante la temporada de incendios. El promedio de la biomasa de todos los sitios fue la biomasa total incorporada para determinado tipo combustible. La suma de las biomasas promedio de cada tipo de combustibles a lo largo del periodo de estudio fue la biomasa total del material depositado durante la temporada de incendios forestales para el área de estudio.

\section{Estimación de las tasas de descomposición}

Para la estimación de la degradación de los combustibles de hojarasca se utilizó el método de bolsas de detritos (Bocock y 
Gilbert, 1957). Se elaboraron 60 bolsas de tela de tul para hojarasca, 29 se dispusieron para contener materiales de $1 \mathrm{~h}$ TR, 20 para 10 h TR y 11 para determinar el peso anhidro de la hojarasca. Cada bolsa contenía $15 \mathrm{~g}$ de hojarasca en peso fresco. En la evaluación de la descomposición de los materiales leñosos de $1 \mathrm{~h}$ y $10 \mathrm{~h}$ TR, se agruparon segmentos de leña a un peso fresco estándar, denominados clase (Tabla 1). Las ramillas eran ajustadas al peso estandarizado recortando extremos, hasta acertar con el peso que correspondiera a la clase. Las bolsas de detritos se colocaron en cuatro sitios del encinar (descritos en el subtítulo anterior).

Para obtener la constante de descomposición, primeramente se calculó el porcentaje de masa remanente (\%MR), como detalla Petit-Aldana et al. (2012), con la siguiente ecuación:

$$
\% \mathrm{MR}=\frac{\mathrm{Mt}}{\mathrm{M} 0}(100)
$$

donde:

$\mathrm{Mt}=$ Peso seco del material remanente al final de la temporada M0 = Peso inicial del material

Se obtuvo el peso seco inicial de las 11 bolsas con hojarasca que no fueron devueltas a campo. El peso seco final fue el peso de cada bolsa de hojarasca terminado el periodo de evaluación y luego de su secado en horno.

TABLA 1. Clases de pesos frescos utilizados para la evaluación de la descomposición de materiales leñosos en un bosque de encino.

\begin{tabular}{cccc}
\hline \multicolumn{2}{c}{ lhTR } & \multicolumn{2}{c}{$10 \mathrm{hTR}$} \\
\hline Clases $(g)$ & No. de piezas & Clases $(g)$ & No. de piezas \\
0.1 & 50 & 0.3 & 8 \\
0.2 & 60 & 0.4 & 8 \\
0.3 & 50 & 0.5 & 6 \\
0.5 & 60 & 1 & 16 \\
1 & 25 & 3 & 16 \\
2 & 4 & 5 & 4 \\
& & 10 & 9 \\
& & 19 & 6 \\
& & 30 & 2 \\
Total & 249 & & 75 \\
\hline
\end{tabular}

Para estimar el peso seco inicial de las muestras de materiales leñosos finos, primeramente con submuestras de ramillas (32 leñas de $1 \mathrm{~h}$ TR, y 12 de $10 \mathrm{~h}$ TR en total), se obtuvieron factores de conversión para obtener el peso seco de los materiales a partir de su peso fresco. Este factor fue 0.832 para los combustibles de $1 \mathrm{~h}$ TR y de 0.874 para los materiales de $10 \mathrm{~h}$ TR. Los factores de conversión se multiplicaron por los pesos frescos de los materialesque se quedaron en campo y, con ello, se infirió su peso seco inicial (M0). También se obtuvo el peso seco de cada ramilla al término de la temporada de incendios $(\mathrm{Mt})$.

Con las masas remanentes de cada bolsa de hojarasca y de cada segmento de leña, se determinó la tasa de descomposición $k$, linealizando el modelo exponencial negativo simple de Olson (1963):

$$
\begin{aligned}
& M t=M 0 e^{-k t} \\
& k t=-\ln \left(\frac{M t}{M 0}\right)
\end{aligned}
$$

donde:

$\mathrm{M} 0=$ masa en el tiempo 0

$\mathrm{Mt}=$ la masa en el tiempo $\mathrm{t}$

$\mathrm{t}=$ tiempo de incubación

$k=$ constante de descomposición

De los valores correspondientes de $k$ por sitio, se obtuvo del promedio por tipo de combustible. El promedio de las constantes de los cuatro sitios fue la tasa de descomposición del área de estudio.

\section{Dinámica de combustibles finos y leñosos finos}

Las constantes de descomposición del encinar se emplearon para estimar la descomposición de las cargas iniciales de los combustibles. La carga que se usó para hacer la comparación fue la determinada por el modelo de Van Wagner (1982), empleando densidades básicas de los materiales del piso forestal. La estimación de la masa remanente al término de la temporada de estiaje se hizo con el modelo de Olson (1963). La estimación de la acumulación de la carga de los combustibles leñosos de $1 \mathrm{~h}$ y $10 \mathrm{~h}$ TR y hojarasca, al final de la temporada, se obtuvo con la masa remanente (masa en el piso forestal), al término de la temporada, más la biomasa de los materiales incorporados. 


\section{Degradación de las ramas de 100 h TR.}

El análisis de la degradación de los combustibles de 100 h TR, se hizo con la densidad básica, a partir de 12 piezas de leñas recolectadas durante la estimación de cargas. De estas leñas se fraccionó una pequeña porción para determinar la densidad básica inicial, el resto se regresó al bosque. Al término de la temporada de evaluación, se recogieron los materiales leñosos y se determinó su densidad básica. El tamaño de los materiales imposibilitó el uso de la báscula para determinar su volumen, por lo que se empleó el método fundamentado en el principio de Arquímides, con desplazamientos de volúmenes de agua en probetas.

\section{Análisis estadísticos}

A cargas de combustibles y tasas de descomposición se les aplicó un Anava, considerando los factores (y niveles): exposición (seca, suroeste; húmeda, noroeste) y dosel (abierto y cerrado). También se aplicó la prueba de comparación de medias de Tukey. Para contenido de humedad, comparación entre materiales firmes y podridos y comparación de cargas iniciales y cargas residuales e incorporación de materiales entre laderas seca y húmeda, se usó la prueba de t de Student. Los análisis estadísticos se hicieron con el programa SAS v. 9 para microcomputadoras.

\section{Resultados}

\section{Contenido de humedad de los combustibles leñosos}

En su mayoría los materiales mostraron de medio a alto grado de pudrición. Durante la evaluación de febrero y mayo, no se encontraron materiales de $100 \mathrm{~h}$ TR. Se hallaron diferencias significativas por el mes en que se realizó la estimación $(\mathrm{P}<$ 0.0001), no así por tiempo de retardo $(\mathrm{P}=0.0853)$ ni para la interacción de ambos factores $(\mathrm{P}=0.2027)$. La prueba de Tukey arrojó diferencias significativas $(\mathrm{p}<0.05)$ entre las medias de noviembre y mayo, así como entre las de noviembre y febrero, pero no así entre febrero y mayo. A mediados y fines de la temporada de incendios se registró el menor $\mathrm{CH}$ de los combustibles leñosos (Tabla 2).

\section{Densidad de los combustibles leñosos}

La prueba $\mathrm{t}$ de Student arrojó diferencias estadísticamente significativas para las medias de las densidades básicas entre materiales firmes y podridos. Se tuvo una $\mathrm{P}<0.0001$, para combustibles con $1 \mathrm{~h}$ y $10 \mathrm{~h}$ TR, y $\mathrm{P}=0.0021$, para aquellos con TR 100 h (Tabla 3).

\section{Carga de combustibles}

La carga estimada para hojarasca fue $38.262 \mathrm{Mg} \mathrm{ha}^{-1}$, en materiales leñosos alcanzó $9.828 \mathrm{Mg} \mathrm{ha}^{-1}$ (con la densidad determinada en laboratorio) y los combustibles vivos tuvieron $7.126 \mathrm{Mg} \mathrm{ha}^{-1}$, con una carga total de $55.217 \mathrm{Mg} \mathrm{ha}{ }^{-1}$. Las cargas de materiales leñosos por las otras variantes usadas para el método de Van Wagner (1982), se muestran en la tabla 4.

TABLA 2. Contenido de humedad (\%) del material leñoso en un bosque de encino, en tres momentos de la temporada de incendios.

\begin{tabular}{llll}
\hline TR & Noviembre & Febrero & Mayo \\
\hline $1 \mathrm{~h}$ & 36.78 & 14.15 & 21.77 \\
$10 \mathrm{~h}$ & 45.78 & 11.66 & 42.23 \\
$100 \mathrm{~h}$ & 31.08 & $\mathrm{~N} / \mathrm{P}$ & $\mathrm{N} / \mathrm{P}$ \\
Promedio general & $37.88 \mathrm{a}$ & $12.90 \mathrm{~b}$ & $32.00 \mathrm{~b}$ \\
\hline
\end{tabular}

$\mathrm{N} / \mathrm{P}=$ no presentes. Medias con diferente letra tuvieron diferencias estadísticamente significativas entre sí.

Comparando las medias de las cargas, con la prueba de t, con respecto a exposición, no se encontraron diferencias significativas. Los valores de P fueron: 0.1284 para el método de Brown (1972), 0.1771 para el de Van Wagner (1982) con determinación de densidad básica en laboratorio y 0.1771 para el mismo pero con densidades obtenidas de la literatura.

\section{Entrada de material combustible}

La hojarasca fue el material que más se incorporó en ambos trimestres. En noviembre-febrero los frutos representaron el segundo mayor aporte (Fig. 3), pero durante febrero-mayo destacaron las leñas. 
TABLA 3. Densidad básica determinada por TR y nivel de descomposición.

\begin{tabular}{lll}
\hline Tiempo de retardo y estado & Densidad $\left(\mathrm{g} \mathrm{cm}^{-3}\right)$ Media & $s$ \\
\hline $1 \mathrm{~h}$ & 0.585 & 0.1133 \\
podridos & $0.472 \mathrm{a}$ & 0.0578 \\
firmes & $0.659 \mathrm{~b}$ & 0.0725 \\
$10 \mathrm{~h}$ & 0.478 & 0.0960 \\
podridos & $0.427 \mathrm{a}$ & 0.0567 \\
firmes & $0.584 \mathrm{~b}$ & 0.0701 \\
$100 \mathrm{~h}$ & 0.412 & 0.0632 \\
podridos & $0.379 \mathrm{a}$ & 0.0433 \\
firmes & $0.464 \mathrm{~b}$ & 0.0549 \\
\hline
\end{tabular}

TABLA 4. Carga de los combustibles leñosos $\left(\mathrm{Mg} \mathrm{ha}^{-1}\right)$ de un bosque de encino, calculada por diferentes métodos.

\begin{tabular}{|c|c|c|c|c|c|c|}
\hline \multirow[t]{2}{*}{ Líneas de intersección } & \multicolumn{2}{|c|}{ Brown (1974) } & \multicolumn{2}{|c|}{$\begin{array}{l}\text { Van Wagner (1982) (densidad } \\
\text { determinada) }\end{array}$} & \multicolumn{2}{|c|}{$\begin{array}{l}\text { Van Wagner (1982) (densidad } \\
\text { de bibliografia) }\end{array}$} \\
\hline & $\begin{array}{l}\text { Ladera } \\
\text { seca }\end{array}$ & $\begin{array}{l}\text { Ladera } \\
\text { húmeda }\end{array}$ & Ladera seca & Ladera húmeda & Ladera seca & Ladera húmeda \\
\hline a & 4.450 & 16.765 & 3.544 & 13.393 & 5.722 & 21.617 \\
\hline b & 10.051 & 7.686 & 7.844 & 5.658 & 12.664 & 9.132 \\
\hline c & 6.409 & 21.381 & 5.071 & 23.466 & 8.185 & 37.876 \\
\hline Carga promedio por sitio & 6.970 & 15.277 & 5.487 & 14.172 & 8.857 & 22.875 \\
\hline $\begin{array}{l}\text { Carga promedio del } \\
\text { encinar }\end{array}$ & & 11.124 & & 9.829 & & 15.866 \\
\hline
\end{tabular}
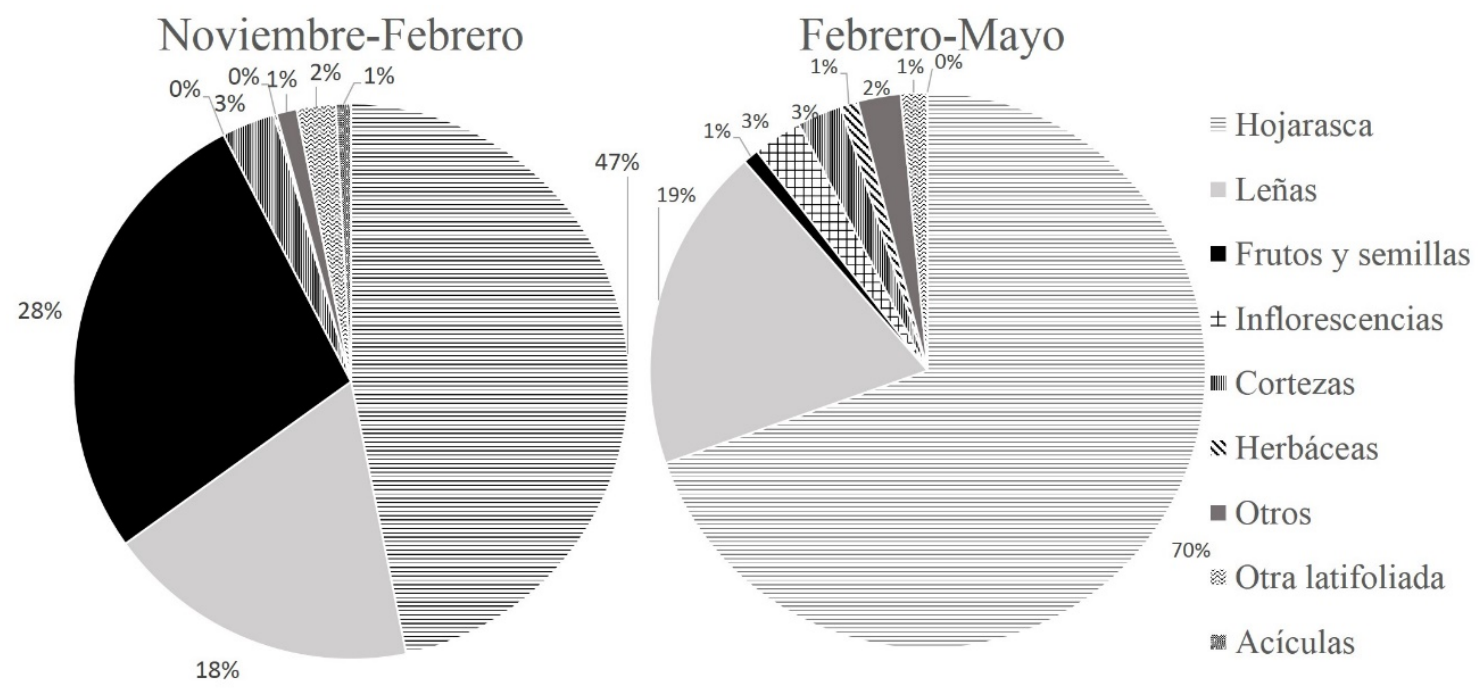

FIGURA 3. Biomasa incorporada (\%) por tipo de material y época. 
La cantidad total de combustible forestal que se incorporó al piso forestal, durante la temporada de incendios fue $40.532 \mathrm{Mg} \mathrm{ha}^{-1}$. La entrada promedio de hojarasca en la ladera húmeda fue 25.55 $\mathrm{Mg} \mathrm{ha}{ }^{-1}$ y en ladera seca alcanzó $25.44 \mathrm{Mg} \mathrm{ha}^{-1}$. No se hallaron diferencias significativas entre ambas laderas (prueba $t$ de Student, $\mathrm{P}=0.9886)$. La entrada promedio de materiales leñosos fue $8.34 \mathrm{Mg} \mathrm{ha}^{-1}$ en la ladera seca y $6.72 \mathrm{Mg} \mathrm{ha}^{-1}$ en la ladera húmeda, sin diferencias estadísticamente significativas entre ambas $(\mathrm{P}=0.5921)$. La entrada indicada de materiales leñosos, corresponde a $1 \mathrm{~h}$ y $10 \mathrm{~h}$ TR, pues no se tuvieron materiales mayores (Tabla 5).

\section{Tasa de descomposición}

La k para hojarasca y materiales leñosos de $1 \mathrm{~h}$ y $10 \mathrm{~h}$ TR durante la temporada seca del año fue, respectivamente: 0.0827, $0.0861 \mathrm{y}$
0.0404. En el Anava de las tasas de descomposición de hojarasca, solamente el factor ladera resultó significativo, no así la cobertura ni la interacción. Los sitios húmedos fueron los que presentaron mayor descomposición (Tabla 6).

En las tasas de descomposición de combustibles con 1 h TR, no se hallaron diferencias significativas para ninguno de los factores estudiados, ni para su interacción. En el caso de los materiales con $10 \mathrm{~h}$ TR, no se hallaron diferencias significativas por ladera ni cobertura forestal, pero sí para la interacción ladera*cubierta $(\mathrm{P}=0.0338)$. Aquí se consideró como covariable el diámetro de los materiales leñosos, pero no resultó significativo $(\mathrm{P}=$ 0.0574). Dicha interacción dejó ver que bajo dosel cerrado no hubo efecto de la ladera en la k, pero bajo dosel abierto resultó mayor la k en la ladera húmeda (Tabla 7 y Fig. 4).

TABLA 5. Cantidad de material combustible $\left(\mathrm{Mg} \mathrm{ha}^{-1}\right)$ incorporado al piso forestal en la temporada seca del año.

\begin{tabular}{cccc}
\hline Tipo de material combustible & $\begin{array}{c}\text { Biomasa incorporada } \\
\text { Noviembre-Febrero }\end{array}$ & $\begin{array}{c}\text { Biomasa incorporada } \\
\text { Febrero-Mayo }\end{array}$ & $\begin{array}{c}\text { Biomasa incorporada } \\
\text { en el estiaje }\end{array}$
\end{tabular}

\begin{tabular}{lccc}
\hline Hojarasca & 5.583 & 19.907 & 25.491 \\
Leña & 2.179 & 5.424 & 7.603 \\
Frutos y semillas & 3.259 & 0.262 & 3.521 \\
& & & \\
Inflorescencias & 0 & 0.82 & 0.82 \\
Cortezas & 0.371 & 0.736 & 1.107 \\
Herbáceas & 0.019 & 0.295 & 0.314 \\
Otros & 0.141 & 0.717 & 0.858 \\
Hojas de otra latifoliada & 0.271 & 0.433 & 0.704 \\
Acículas & 0.104 & 0.011 & 0.115 \\
Biomasa total incorporada & 11.926 & 28.606 & 40.532 \\
\hline
\end{tabular}

TABLA 6. Tasa de descomposición de hojarasca.

\begin{tabular}{|c|c|c|c|c|c|}
\hline Factor & Nivel & $P$ & $k$ & $k$ (día) & Masa remanente (\%) \\
\hline \multirow{2}{*}{ Ladera } & Húmeda & \multirow{2}{*}{0.0202} & 0.0983a & 0.00061 & 90.8 \\
\hline & Seca & & $0.0672 b$ & 0.00042 & 93.5 \\
\hline \multirow{2}{*}{ Cobertura } & Abierta & \multirow{2}{*}{0.1783} & $0.0736 a$ & 0.00046 & 93 \\
\hline & Cerrada & & 0.0919a & 0.00057 & 91.3 \\
\hline Ladera*cobertura & & 0.3083 & & & \\
\hline
\end{tabular}


Madera y Bosques vol. 24, núm. 2, e2421467 Verano 2018

TABLA 7. Tasa de descomposición para materiales con 1 h y 10 h TR.

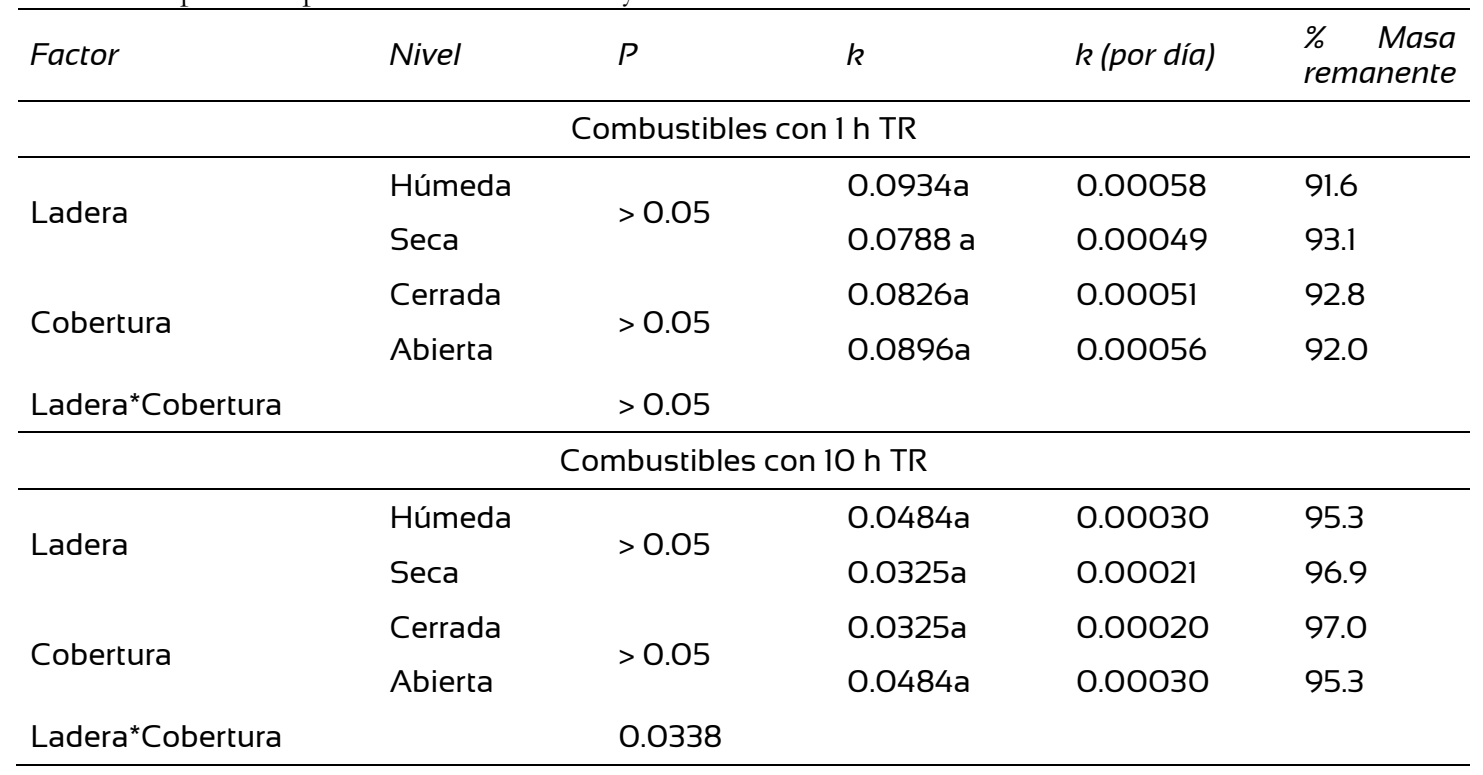

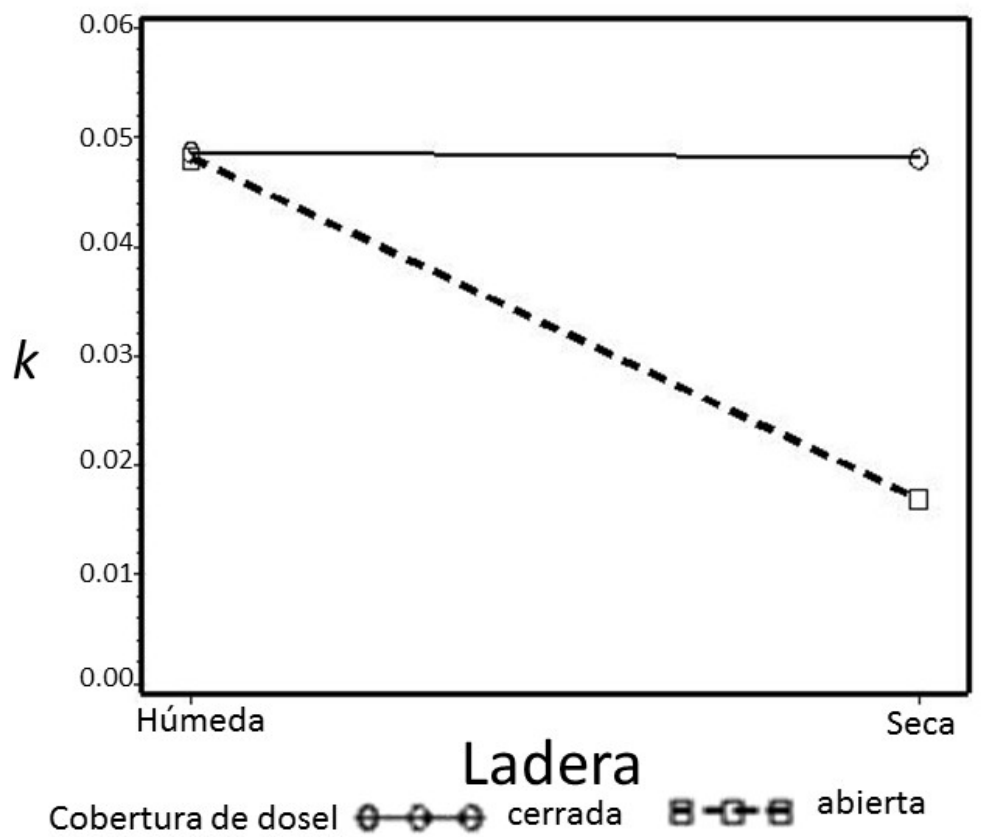

FIGURA 4. Interacción ladera y cobertura para materiales leñosos con TR $=10 \mathrm{~h}$.

Los combustibles con $100 \mathrm{~h}$ TR, tuvieron densidades medias iniciales (y desviación estándar) de 0.452 (0.0551) y final de 0.373 (0.0486), lo que representó una reducción de 17.5\%, así como una $\mathrm{k}$ de 0.1932 .

\section{Dinámica de combustibles}

Al descontar el material que se descompuso y sumar el que se incorporó, resultó un aumento en la carga de combustibles leñosos y de hojarasca durante la temporada de incendios (Tabla 8). 
TABLA 8. Dinámica de combustibles durante la temporada de incendios.

\begin{tabular}{|c|c|c|c|c|c|c|c|}
\hline Tipo de combustible & $\begin{array}{l}\text { Carga inicial } \\
\qquad\left(M g h a^{-1}\right)\end{array}$ & $K$ & $\begin{array}{l}\text { Descomposición } \\
\left(\mathrm{Mg} \mathrm{ha}^{-1}\right)\end{array}$ & $\begin{array}{l}M R \\
\left(M g h a^{-1}\right)\end{array}$ & $\begin{array}{l}\text { Entrada } \\
\left(M g h a^{-1}\right)\end{array}$ & $\begin{array}{l}\text { Acumulación } \\
\left(M g h a^{-1}\right)\end{array}$ & $\begin{array}{l}\text { Incremento } \\
(\%)\end{array}$ \\
\hline Leñas 1 y $10 \mathrm{~h}$ TR & 3.60 & 0.0632 & 0.220 & 3.380 & 7.603 & 10.983 & 205.0 \\
\hline Hojarasca & 38.26 & 0.0827 & 3.035 & 35.225 & 25.491 & 60.716 & 58.7 \\
\hline
\end{tabular}

MR = masa residual a la descomposición.

\section{DISCUSIÓN}

\section{Contenido de humedad de los materiales leñosos}

El menor contenido de humedad conforme avanza la temporada de incendios, se relaciona con la escasez de lluvias durante tal periodo. La falta de diferencias en contenido de humedad entre materiales con diferente TR, puede deberse al contacto con el suelo, que pierde su humedad más lentamente y que también intercambia humedad con los combustibles leñosos, así como a la presencia de elevada humedad relativa o precipitación esporádicas.

\section{Densidad de los combustibles leñosos}

La tendencia que muestra la densidad básica de los combustibles leñosos, es decreciente a mayores tiempos de retardo, como refieren Brown (1974) para coníferas norteamericanas y MorfinRíos et al. (2012) para bosques de pino-encino del occidente de México. Las altas densidades básicas en los materiales de $1 \mathrm{~h}$ de tiempo de retardo pueden ser una adaptación para contar con mayor resistencia, pese a su delgadez (Rodríguez-Trejo, 2015). Esta variación puede deberse también a que una vez terminado el proceso de abscisión, las ramillas con $1 \mathrm{~h}$ de TR caen, mientras que las ramas más gruesas se mantienen adheridas al árbol donde continúan su proceso descomposición y, al incorporarse al piso forestal, ya llevan cierto grado de pudrición. La densidad básica estuvo inversamente relacionada con el tiempo de retardo. Como se esperaba, los materiales podridos tienen menor densidad.

\section{Carga de los combustibles leñosos}

La variante del método de Van Wagner (1982), con la determinación de la densidad básica de los materiales leñosos en laboratorio, es la más precisa, resulta una carga $11.63 \%$ menor que con la utilización del método de Brown para coníferas y 42.63\% menos que con densidades básicas de la literatura. Por ende, cualquiera de las dos opciones diferentes a la determinación de densidad básica, sobreestimó la carga de combustibles leñosos.

No se halló un efecto significativo por método de determinación de la carga de materiales leñosos, la alta variabilidad de cargas en cada sitio influyó en ello. Por otra parte, la determinación de la densidad de los combustibles leñosos en laboratorio permite obtener densidades reales de materiales que se hallan en diferentes niveles de descomposición, cosa que no se logra captar si se trabaja con densidades obtenidas de la literatura para materiales sin descomposición. Las determinaciones más precisas, de laboratorio, permitirán calcular cargas más reales y mejorar todo tipo de estimaciones, como emisiones o comportamiento del fuego.

\section{Entrada de combustibles}

La entrada de 40.5 Mg ha-1 de combustibles es alta, debido a que esta evaluación corresponde a la temporada seca, época en que hay mayor incorporación de materiales (Martínez-Yrízar y Sarukhán, 1993), así como al comportamiento semicaducifolio de las especies. En bosques secos de eucalipto en Australia, las entradas de combustible más altas se estimaron en $1.3 \mathrm{Mg}$ ha-1 y 2.9 $\mathrm{Mg}$ ha-1 durante la temporada de incendios veraniegos (Gould et al., 2007). Por otra parte, las especies de encino estudiadas forman masas de etapas sucesionales iniciales (ZavalaChávez, 2007) y, conforme la sucesión avanza, tienden a acumular materiales orgánicos en el piso forestal (Odum y Barrett, 2006). Más aun, los vendavales que se presentaron en el área de estudio acumularon materiales muertos. Sin embargo, otros autores han encontrado datos similares. Por ejemplo, en ecosistemas de 
Estados Unidos la incorporación anual de ramillas de $1 \mathrm{~h}$ TR, para una sola especie, se estimó en $38 \mathrm{Mg}$ ha-1 (Agee, Wakimoto y Biswell, 1976).

Esto es relevante, pues Scott, Bowman, Bond, Pyne y Alexander (2014) aseveran que la tasa de propagación del frente del fuego es directamente proporcional a la carga de combustibles finos $(T R=1 \mathrm{~h})$ consumidos. Es necesario hacer la estimación de entrada de biomasa en la temporada de lluvias, debido a que la lluvia, granizos y los fuertes ventarrones, pueden incrementar la incorporación de ramas al piso forestal. Asimismo, por las mayores temperaturas y humedad, la descomposición es mayor durante tal época.

\section{Tasas de descomposición}

Las tasas de descomposición obtenidas son bajas si se comparan con las de los bosques deciduos, con Q. alba L., en Carolina del Norte, donde dicha variable osciló entre 0.17 año-1 y 0.25 año-1 para los combustibles leñosos finos (Fasth, Harmon y Sexton, 2011). Una de las causas de los valores bajos de $\mathrm{k}$, se atribuye a que la investigación se realizó únicamente durante la temporada seca del año, ya que la descomposición se efectúa más rápidamente durante la temporada de lluvia (Álvarez y Harmon, 2003).

El que los combustibles de $10 \mathrm{~h}$ TR presentaran menores tasas de descomposición se debió al efecto de la relación superficie/volumen de los materiales. Las ramillas de $1 \mathrm{~h}$ TR tienen una mayor superficie de contacto con los agentes degradadores bióticos y abióticos, en comparación con los materiales de 10 h TR. De acuerdo con Herrmann, Kahl y Bauhus (2015), el grosor de los combustibles de 10 h TR, les confirió mayor resistencia a la descomposición. Müller-Using y Bartsch (2009) anotan constantes de descomposición bajas (0.089) (y por ende tasa de descomposición más lenta) en materiales leñosos gruesos, comparadas con 0.178 para ramas con diámetros entre 1 $\mathrm{cm}$ y $10 \mathrm{~cm}$, y 0.22 para ramillas menores a $1 \mathrm{~cm}$ de diámetro, en Fagus sylvatica L. En este caso, tal variación hizo que la descomposición de ramillas menores a $1 \mathrm{~cm}$ ocupara cuatro años, un cuarto del tiempo de los materiales menores de $10 \mathrm{~cm}$ (16 años).

Otro factor que probablemente intervino en la diferencia de tasas de descomposición, fue el nivel de degradación de los materiales al incorporarse al suelo, pues la tasa de descomposición es mayor al inicio del proceso de putrefacción y con el tiempo se torna lenta (Álvarez-Sánchez y Harmon, 2003; Müller-Using y Bartsch, 2009). Los materiales firmes que se incorporan a la cama de combustible, al principio presentan una acelerada descomposición al entrar en contacto con los microorganismos descomponedores y humedad del suelo, que favorece a estos últimos, mientras los árboles en pie, incluyendo las ramas muertas retenidas en el árbol, mantienen una lenta descomposición en el aire (Hérault et al., 2010) y, una vez que caen, su descomposición continúa pero no con la misma velocidad. Las ramillas de $1 \mathrm{~h}$ TR, una vez terminado su proceso de abscisión caen al piso, mientras que algunas ramillas de $10 \mathrm{~h}$ TR permanecen adheridas al árbol. La mayor tasa de descomposición en la ladera húmeda, con respecto a la seca, coincide con los resultados de Bonilla-Padilla, Rodríguez-Trejo, Borja de la Rosa, Cíntora-González y SantillánPérez (2013) para Q. crassifolia Humb. \& Bonpl. en Chignahuapan, Puebla.

\section{Descomposición de los combustibles con 100 h TR}

Comparado con datos anuales indicados en diferentes investigaciones, el decremento en la densidad de los materiales de $100 \mathrm{~h}$ TR es alto para el tiempo de evaluación. Fue similar a lo señalado para un año en bosque de latifoliadas del sureste de los Apalaches, en Carolina del Norte (Mattson, Swank y Waide, 1987) y luego de dos años en bosques de eucaliptos y plantaciones de coníferas en Australia (Mackensen y Bauhus, 2003). En relación con el primer caso, la explicación estriba en que la temperatura es menor en Carolina del Norte y, para el segundo, con clima mediterráneo, la menor precipitación y humedad en esta zona de Australia ralentizan la descomposición.

Asimismo, los encinos forman duramen en la madera, también por ello se espera que los combustibles de $100 \mathrm{~h}$ TR presenten una menor tasa de descomposición. Tainter y McMinn (1999), encontraron que la densidad básica de la albura, evaluada en tres condiciones ambientales, disminuyó conforme pasó el tiempo, pero no fue así para el duramen.

La elevada densidad de la madera de Quercus le confiere resistencia a la descomposición y, debido a ello, se tienen lentas tasas en dicho proceso. Sin embargo, en este trabajo se obtuvieron mayores tasas (0.1932), una posible causa pudo ser 
que los materiales leñosos de los sitios de muestreo eran viejos (tenían baja densidad inicial), aunque no exhibían una marcada pudrición.

\section{Dinámica de combustibles}

Al final de la temporada de estiaje se estimó un incremento de $204.98 \%$ en materiales de 1 h y 10 h TR y $58.68 \%$ para hojarasca, con respecto a la carga inicial. Lo anterior aumenta el peligro de incendio forestal, en su componente de carga de combustibles. Conforme a estadísticas de la Comisión Nacional Forestal [Conafor] (2016), es a fines de la temporada seca que se presentan más incendios, lo que a su vez también representa mayor riesgo en esa época.

\section{CONCLUSIONES}

Las distintas metodologías usadas para la estimación de los combustibles, arrojan diferencias numéricas en la estimación de la carga. A pesar de que el uso de las densidades básicas de los materiales leñosos determinadas en la cama de hojarasca resulta la menor, ésta debe considerarse como la carga del encinar de estudio, pues se hizo con base en las propiedades de los combustibles forestales del sitio. Se considera que la elevada variabilidad de carga entre sitios de muestreo de cada tratamiento no dejó ver las potenciales diferencias entre métodos para obtener la carga.

La densidad básica estimada es menor a la densidad básica registrada, porque esta última se obtiene a partir de materiales firmes, sin descomposición; los materiales presentes en el mantillo del bosque tienen ya diferentes grados de descomposición.

En Quercus, los materiales leñosos finos son más densos que los materiales gruesos. La tendencia descendente de la densidad básica a mayor TR, puede deberse a que las ramillas delgadas deben ser más resistentes para realizar sus funciones de soporte y mantenerse en el árbol por el tiempo suficiente para realizar sus funciones.

La mayor incorporación de material combustible (y su baja humedad), de febrero a mayo, confieren mayor peligro de incendio.
Las ramillas de $1 \mathrm{~h}$ y 10 h TR son las más dinámicas en comparación con los combustibles más gruesos, estos últimos no fueron observados el periodo de evaluación de incorporación.

La hojarasca presenta la mayor tasa de descomposición durante la época seca del año y es también la que mayor carga aporta al piso forestal.

Por tratarse de una masa no clímax, los materiales se están acumulando y continuarán haciéndolo los próximos años, por la mayor velocidad de incorporación que la de descomposición.

\section{RECONOCIMIENTOS}

Al Conacyt y a la UACH.

\section{REFERENCIAS}

Adame-Martínez, S., Oropeza-Mota, J. L., Martínez-Ménez, M. R., Santoyo-Cortés, V. H., \& Ramírez-Guzmán, M. E. (2000). Evaluación económica del proyecto de rehabilitación de suelos en la cuenca del río Texcoco. Agrociencia, 34, 509-521.

Agee, J. K., Wakimoto, R. H., \& Biswell, H. H. (1976). Fire and fuel dynamics of Sierra Nevada conifers. Forest Ecology and Management, 1, 255-265. doi: 10.1016/03781127(76)90030-X

Álvarez-Sánchez, J., \& Harmon, M. E. (2003). Descomposición de hojarasca: hojas y madera. En J. Álvarez-Sánchez, \& E. Naranjo-García (Eds.), Ecología del suelo en la selva tropical bumeda de México (pp. 108-122). Ciudad de México: Universidad Nacional Autónoma de México.

Augustine-Molumeli, P., Espinosa-Hernández, V., Ehsan, M., Benedicto-Valdez, S., Ojeda-Trejo, E., Cetina-Álcala, V. M., \& Santamaría-Delgado, K. (2008). Lupines-invaded pine forest and cultivated scrublands in volcanic ash soils in Mexico: Dry-sieved aggregation and instability indices. International Journal of Botany, 4(4), 390-405.

Bocock, K. L., \& Gilbert, O. J. W. (1957). The disappearance of leaf litter under different woodland conditions. Plant and Soil, 9(2), 179-185. doi: 10.1007/BF01398924

Bonilla-Padilla, E., Rodríguez-Trejo, D. A., Borja de la Rosa, A., Cíntora-González, C., \& Santillán-Pérez, J. (2013). Dinámica de combustibles en rodales de encino-pino de Chignahuapan, Puebla. Revista Mexicana de Ciencias Forestales, 4(19), 20-33.

Brown, J. K. (1974). Handbook for Inventorying Downed Woody Material. General Technical Report INT-16. USDA Forest Service. Ogden, Utah. 
Byram, G. M., Fons, W. L., Sauer, F. M., \& Arnold, R. K. (1952). Thermal properties of forest fuels (Interim Technical Report 404 for AFSWP). Berkeley, California: Division of Fire Research. U.S. Department of Agriculture.

Comisión Nacional Forestal [Conafor]. (2016). Reporte de incendios forestales. Inédito.

Fasth, B. G., Harmon, M. E., \& Sexton, J. (2011). Decomposition of fine woody debris North Carolina. Journal of the Torrey Botanical Society, 138(2), 192-206.

Flores-Garnica, J. G., Rodríguez-Chávez, O. G., MeléndezGómez, M., \& Rosas-Aceves, O. G. (2008). Sitios de muestreo para monitoreo de combustibles forestales (SIMMCOF). Guadalajara, Jalisco, México: Instituto Nacional de Investigaciones Forestales, Agrícolas y Pecuarias.

Gould, J. S., MtCaw, W. L., Cheney, N. P., Ellis, P. F., Knight, I. K., \& Sullivan, A. L. (2007). Project Vesta-Fire in dry Eucalypt forest: fuel structure, fuel dynamics and fire behaviour. Perth, WA: Ensis-CSIRO, Canberra ACT, Departmen of Environmen and Conservation.

Hérault, B., Beauchêne, J., Muller, F., Wagner, F., Baraloto, C., Blanc, L., \& Martin, J. M. (2010). Modeling decay rates of dead wood in a neotropical forest. Oecologia, 164(1), 243251. doi: 10.1007/s00442-010-1602-8

Herrmann, S., Kahl, T., \& Bauhus, J. (2015). Decomposition dynamics of coarse woody debris of three important central European tree species. Forest Ecosystems, 2(1), 27. doi: 10.1186/s40663-015-0052-5

Hyde, J. C., Smith, A. M. S., Ottmar, R. D., Alvarado, E. C., \& Morgan, P. (2011). The combustion of sound and rotten coarse woody debris: a review. International Journal of Wildland Fire, 20, 163-174.

Instituto Nacional de Estadística, Geografía e Informática [Inegi]. (2005). Censo de población y vivienda. Principales resultados por localidad. México. Inegi.

Karberg, N. J., Scott, N. A., \& Giardina, C. P. (2008). Methods for estimating litter decomposition. En C. M. Hoover (Ed.). Field Measurements for Forest Carbon Monitoring (pp. 103-111). Nueva York: Springer.

Landsberg, J. J., \& Gower, S. T. (1997). Applications of physiological ecology to forest management. San Diego: Academic Press.

Le Quéré, C., Andres, R. J., Boden, T., Conway, T., Houghton, R. A., House, J. I., Marland, G., Peters, G. P., van der Werf, G. R., Ahlström, A., Andrew, R. M., Bopp, L., Canadell, J. G., Ciasis, P., Doney, S. C., Enright, C., Friedlingstein, P., Huntingford, C., Kain, A. K., Jourdain, C., Kato, E., Keeling, R. F., Klein Goldewijk, K., Levis, S., Levy, P., Lomas, M., Poulter, B., Raupach, M. R., Schwinger, J., Sitch, S., Stocker, B. D., Viovy, N., Zaehle, S., \& Zeng, N.
(2013). The global carbon budget 1959-2011. Earth System Science Data, 5(1), 165-185. doi: 10.5194/essd-5-165-2013

Mackensen, J., \& Bauhus, J. (2003). Density loss and respiration rates in coarse woody debris of Pinus radiata, Eucalyptus regnans and Eucalyptus maculata. Soil Biology and Biochemistry, 35(1), 177-186. doi: 10.1016/S0038-0717(02)00255-9

Martínez-Yrízar, A., \& Sarukhán, J. (1993). Cambios estacionales del mantillo en el suelo de un bosque tropical caducifolio y uno subcaducifolio en Chamela, Jalisco, México. Acta Botánica Mexicana, 21, 1-6.

Mattson, K., Swank, W., \& Waide, J. (1987). Decomposition of woody debris in a regenerating, clear-cut forest in Southern Appalachains. Canadian Journal of Forest Research, 17, 712721. doi: 10.1017/CBO9781107415324.004

Morfin-Ríos, J. E., Jardel Peláez, E. J., Alvarado Celestino, E., \& Michel-Fuentes, J. M. (2012). Caracterización y cuantificación de combustibles forestales. Comisión Nacional Forestal-Universidad de Guadalajara. Guadalajara, Jalisco, México.

Müller-Using, S., \& Bartsch, N. (2009). Decay dynamic of coarse and fine woody debris of a beech (Fagus sylvatica L.) forest in Central Germany. European Journal of Forest Research, 128(3), 287-296. doi: 10.1007/s10342-009-0264-8

Odum, E. P., \& Barrett, G. W. (2006). Fundamentos de Ecología. México: CENCAGE.

Olson, J. S. (1963). Energy storage and the balance of producers and decomposers in ecological systems. Ecology, 44(2), 322331.

Ordóñez-Díaz, J. A. B., Galicia-Naranjo, A., Venegas-Mancera, N. J., Hernández-Tejeda, T., Ordóñez-Díaz, M. J., \& Dávalos-Sotelo, R. (2015). Densidad de las maderas mexicanas por tipo de vegetación con base en la clasificación de J. Rzedowski: compilación. Madera y Bosques, 21(Núm. esp.), 77-126. doi: 10.21829/myb.2015.210428

Petit-Aldana, J., Uribe-Valle, G., Casanova-Lugo, F., SolorioSánchez, J., \& Ramírez-Avilés, L. (2012). Descomposición y liberación de nitrógeno y materia orgánica en hojas de Leucaena leucocephala (Lam.) de Wit, Guazuma ulmifolia Lam. y Moringa oleifera Lam. en un banco mixto de forraje. Revista Chapingo Serie Ciencias Forestales y del Ambiente, XVIII(1), 525. doi: 10.5154/r.rchscfa.2011.03.025

Rodríguez-Trejo, D. A. (2015). Incendios de vegetación: su ecología, manejo e historia (Vol. 2). Guadalajara, Jalisco, México: Biblioteca Básica de Agricultura.

Romero-Rangel, S., Rojas-Zenteno, E. C., \& Aguilar-Enríquez, M. de L. (2002). El Género Quercus (Fagaceae) en el Estado de México. Annals of the Missouri Botanical Garden, 89(4), 551-593. 
Sandberg, D. V., Ottmar, R. D., \& Cushon, G. H. (2001). Characterizing fuels in the 21 st century. International Journal of Wildland Fire, $10(3$ and 4), 381-387. doi: 10.1071/WF01036

Scott, A. C., Bowman, D. M. J. S., Bond, W. J., Pyne, S. J., \& Alexander, M. E. (2014). Fire on Earth: An introduction. Chichester: Wiley Blackwell.

Tainter, F. H., \& McMinn, J. W. (1999). Early deterioration of coarse woody debris. Tenth Biennial Southern Silvicultural Research Conference, 232-237.

Van Wagner, C. E. (1968). The line intersect method in forest fuel sampling. Forest Science, 14(1), 20-26.

Van Wagner, C. E. (1982). Practical aspects of the line intersect method. Canadian Forest Service. Chalk River, Ontario, Canada.

Zavala-Chávez, F. (2007). Guía de los encinos de la Sierra de Tepotztlán, México. Chapingo, Edo. de México: Universidad Autónoma Chapingo.
Manuscrito recibido el: 27 de noviembre de 2016

Aceptado el: 13 de febrero de 2018

Publicado el: 22 de agosto de 2018

Este documento se debe citar como:

Martínez-Villa, J. L., Rodríguez-Trejo, D. A, Borja-de la Rosa, M. A. M, Rodríguez-Yam, G. A. (2018). Propiedades físicas y dinámica de los combustibles forestales en un bosque de encino. Madera y Bosques, 24(2), e2421467. doi: 10.21829/myb.2018.2421467

Madera y Bosques por Instituto de Ecología, A.C. se distribuye bajo una Licencia Creative Commons Atribución-NoComercialCompartirlgual 4.0 Internacional. 